\title{
Emergence of Three-Dimensional Printing Technology and Its Utility in Spine Surgery
}

\author{
Akshay Gadia ${ }^{1}$, Kunal Shah ${ }^{2}$, Abhay Nene ${ }^{1}$ \\ ${ }^{1}$ Department of Spine, Wockhardt Hospital, Mumbai, India \\ "We Are Spine" Centre, Mumbai, India
}

In the last decade, spine surgery has advanced tremendously. Tissue engineering and three-dimensional (3D) printing/additive manufacturing have provided promising new research avenues in the fields of medicine and orthopedics in recent literature, and their emergent role in spine surgery is encouraging. We reviewed recent articles that highlighted the role of 3D printing in medicine, orthopedics, and spine surgery and summarized the utility of 3D printing. 3D printing has shown promising results in various aspects of spine surgery and can be a useful tool for spine surgeons. The growing research on tissue bioengineering and its application in conjunction with additive manufacturing has revealed great potential for tissue bioengineering in the treatment of spinal ailments.

Keywords: Three-dimensional printing; Computer-aided designing; Spine surgery; Orthopedics

\section{Introduction}

Three-dimensional (3D) printing or additive manufacturing is a technique that uses computer-aided design (CAD) files to manufacture physical objects layer-bylayer additively without using molds [1]. Although 3D printing technology has been popular since the 1980s, it has recently received much attention because advances in the technology have made it possible to produce almost anything, from prototype models of cars to homes [2]. The application of $3 \mathrm{D}$ printing in medicine has grown exponentially in the last decade because of decreased cost, computer engineering, and ever-growing applications. There are individual variances in human anatomy; the use of $3 \mathrm{D}$ printing has allowed surgeons to plan customized surgeries. The current practice and teaching of medicine is changing slowly but steadily because of the use of biomodels and biological tissue engineering of implantable prosthetics [3]. Although the traditional approach to medicine has always been restricted to using timetrial treatment methods, the current trends involve using patient-specific and customized care. The relevance of 3D printing in medicine and its use in orthopedics have been well described in the recent literature. In this article, we provide an overview of the application of 3D printing in spine surgery and in the treatment of spinal diseases.

\section{Process of Three-Dimensional Printing}

For manufacturing physical objects using $3 \mathrm{D}$ printing, 3D CAD files are used [4]. First, an image is acquired using a multidetector computed tomography (CT) scan. Then, a $3 \mathrm{D}$ image simulating the required object is created on a computer. Using software, digital cross sections are made

Received Oct 24, 2017; Revised Nov 2, 2017; Accepted Nov 2, 2017

Corresponding author: Kunal Shah

"We Are Spine" Centre, Aarav Polyclinic, 101 Excel Arcade, Opposite Telephone Exchange, LBS Road, Ghatkopar West, Mumbai 400086, India

Tel: +91-9930731911, E-mail: orthokunal@yahoo.com 
into the CAD file. This data is transferred to a $3 \mathrm{D}$ printer for manufacturing the object layer-by-layer additively by depositing polyurethane powder without using molds. Various materials, such as ceramic, resin, metal, plastic, polyurethane, or glass, can be used to manufacture 3D models [5]. The layers are fused together using various technologies such as sintering with laser or electronic beams that harden the powder to make a base for the new object. This process depends on the technical specifications of the 3D printing mechanism being used and the desired final product. Other 3D printing methods include selective laser melting, laser sintering, fused deposition modeling, stereolithography, laminated object manufacturing, and fused filament fabrication [5].

\section{Applications in Medicine}

The applications of 3D printing in medicine include customized hearing aids, patient-specific rehabilitation tools and orthoses and customized implants. One of the most common uses of $3 \mathrm{D}$ printing technology is to produce customized bone prosthetics using high-definition CT scans. One important application of this technology is in maxillofacial surgery, wherein surgeons face difficulty due to the complicated native anatomy of the maxillofacial region. The first successful customized 3D-printed mandibular titanium prosthesis was implanted in an 83-yearold female at University of Belgium [6]. The manufacturing technique used a laser to melt successive thin layers of titanium powders to build the prosthesis using a digital 3D image. In 2013, Food and Drug Administration (FDA) granted approval for producing polyethylethylketone (PEEK) skull implants [7]. PEEK has a dual advantage of having a near equivalent structure to that of titanium and elasticity similar to that of cancellous bone [8]. The use of custom-made porous titanium or PEEK prosthetics may be particularly interesting to spine surgeons because of its capacity to serve as a scaffold for bone ingrowth. FDA also approved the implantation of a 3D-printed tracheal splint in an infant, born with tracheobronchomalacia $[9,10]$.

$3 \mathrm{D}$ printing is also used to create models for practicing, teaching, and surgical planning in complicated pathologies and anatomy. Neurosurgeons often encounter complicated anatomy. The intricate and sometimes obscure structural relationships between cranial nerves, blood vessels, and the structure of the skull base cannot be accurately defined on two-dimensional CT scans. Fur- thermore, the lack of accurate understanding of this complicated anatomy may lead to devastating complications perioperatively. Therefore, for teaching and presurgical planning as well as for understanding this intricate anatomy preoperatively, customized 3D-printed models should be made [11].

Hepatobiliary surgeons have used 3D printing technology for planning liver transplants. Replicas of a patient's organ are used to determine the amount of carving required to fit the donor's liver into the recipient's abdomen. The 3D models used were made of partially transparent low-cost acrylic resin or polyvinyl alcohol having water content and texture similar to those of living tissues, which allowed surgical blades to cut into the models more realistically [12].

Cardiothoracic surgeons are also interested in using 3D printing for patient care. Recently, a team of surgeons led by Professor Jeffery L. Port at Presbyterian Weill Cornell Medical Center, New York, performed the first surgery in the United States to replace the sternum and part of the rib cage in a 20 -year-old female with a customized 3D-printed implant. This implant was used to fill the void caused by the resection of a malignant chondrosarcoma of the sternum [13].

The most intriguing, advanced, and exciting application of $3 \mathrm{D}$ printing in medicine is tissue engineering, which involves the formation of inert scaffolds for biological ingrowth of cells in vivo for transplantation. Bioprinting is the process of $3 \mathrm{D}$ printing all individual components to form a tissue. There are far-reaching applications for combining degradable or allogeneic scaffolding with cellular bioprinting to create customized biological prosthetics that have great potential to serve as transplantable replacement tissue. Bioprinting technology shows much promise for tissue engineering because it enables precise placement of cells and biomaterials to construct a tissue that is similar in configuration as native tissue. Murphy and Atala [14] have demonstrated the manufacturing of complete and functional kidneys using 3D bioprinting. The kidneys were made with a mixture of human cells and a gel-like biodegradable scaffold [14]. They also developed bioprinters that are able to print various cell types ranging from vascular cells to stem cells $[15,16]$. Ringeisen et al. [17] from the University of Texas at El Paso developed a method of printing fat tissue with an expectation to use it eventually for women undergoing partial mastectomy and breast lumpectomy. 


\section{Use of Three-Dimensional Printing in Orthopedics}

3D printing is steadily finding a foothold in orthopedic surgery because of its vast application to resolve the unmet needs in orthopedics in both operative and nonoperative patient care. Areas to be addressed include bone defects, spinal arthrodesis, osteochondral injury, periprosthetic joint infection, customized prostheses following amputation, and customized exoskeletons.

$3 \mathrm{D}$ printing is useful in complex trauma cases. The $3 \mathrm{D}$ printed models provide a visual and tactile aid in conceptualizing complex fracture patterns [3]. Preoperative review of the $3 \mathrm{D}$ model can allow the surgeon to anticipate intraoperative difficulties and select an optimal surgical approach and indicate the need for specific equipment. Challenging pelvic fractures provide an example of these concepts $[18,19]$. The management of bone defects following a compound trauma or an infectious non-union is a challenging task. The main advantage of using 3D printing technology is the capability to fabricate inert bone spacers (implants) of complex external shapes and internal structures, including the capacity to create porous structure for weight reduction, tailoring stiffness, and osteointegration. A 3D-printed spacer accurately fits into the defect site because it is developed using patient-specific data. Moreover, implants designed with specific geometry and material help avoid the effects of stress shielding because they can closely match the stiffness of bone [20]. Ulbrich et al. [21] propagated 3D-printed models that were manufactured via rapid prototyping as a tool for preoperative planning and surgeon-to-patient demonstration for better understanding of the pathology and procedure to be performed. They also suggested that it may improve accuracy, reduce surgical duration and morbidity, and improve overall surgical outcome.

The use of tissue engineering in the treatment of osteochondral injuries involves scaffold-only and cell-only techniques. These two techniques represent the two ends of a continuous spectrum, wherein many surgeons employ the combination of cells, growth factors, and scaffolds to facilitate tissue regeneration [22]. Cui et al. [23] used thermal-based inkjet printing technology to produce a bioartificial cartilage.

The application of $3 \mathrm{D}$ printing is growing rapidly in the field of prosthetic limbs. Because of the ever-growing burden of congenital anomalies and loss of limbs through accidents, war, and diseases, prosthetics have become an important part of health care. 3D-printed prosthetics have an advantage of resembling the near normal appearance of a patient's specific body part because they are produced from patient-specific data. Patients who undergo upper limb amputation have many options, such as a nonfunctional cosmetic hand and a neuroprosthetic arm [24]. Modern lower limb prosthesis can facilitate the physical function at a level that is almost indistinguishable from that of a normal person with intact lower limbs [24]. The conventional process of manufacturing these prostheses would involve the formation of a mold followed by minute adjustments according to individual needs, which would be time consuming and cause inconvenience to the patient. However, the use of $3 \mathrm{D}$ printing is more convenient and provides a shorter turnaround time.

Standard therapy for deep infections in joint replacement involves prosthesis removal, debridement, implantation of an antibiotic impregnated spacer, and replacement with a definitive prosthesis following eradication of the infection. Cement spacers are typically produced intraoperatively [25]; this may take time and result in cement of suboptimal dimensions and constrained use of heat-stable antibiotics. 3D printing allows for manufacturing spacers that may be used over the shelf and provides sustained release of heat-sensitive antibiotics [26].

\section{Use of Three-Dimensional Printing in Spinal Care}

$3 \mathrm{D}$ printing in spine surgery has various uses such as designing patient-specific spinal orthoses and producing customized spinal implants. 3D-printed tissue engineering has recently gained the attention of researchers and provided excellent results in complex surgeries. Additive manufacturing is now widely used to produce customized spinal implants. In 2009, at Peking University Hospital, Beijing, Xiu et al. [27] was the first to develop titanium spinal implants using electron-beam melting 3D printers. It was noted that $3 \mathrm{D}$-printed implants were superior to nonprinted implants because of their ability to print specific anatomically-based structures. Because of the relative ease of creating porous material, 3D-printed implants can serve as a scaffold for the ingrowth of bone. Human clinical trials are underway and have shown positive preliminary results [27]. 


\section{Spinal Orthoses}

Bagaria et al. [28] explored the utility of 3D printing in designing customized orthoses for patients with spinal deformity. They believed that the conventional braces that were made out of molds had a major noncompliance because of multiple reasons. CAD software was used to create a patient-specific template, over which the brace was made layer-by-layer. This brace had significant compliance with the patient. Global advancement in the technology would allow the widespread use of this technique cutting across all social structures.

\section{Spine Biomodels}

D'Urso et al. [29] proposed the use of biomodeling as an aid to spinal instrumentation. Acrylate biomodels were designed by rapid prototyping using 3D CT scans of patients. Trajectory pins were drilled in these biomodels to preoperatively simulate the surgery. Acrylate drill guides were designed using these trajectory pins and biomodels that were used intraoperatively to place the actual pedicle screws [29]. Because the biomodels and drill guides were made up of acrylate, they were sterilized and used intraoperatively to provide surgeons a visual cue for placing the pedicle screw. CT scans were performed in all patients for postoperatively confirming the position of screws. The authors also found that the use of biomodels in preoperative counseling improved the content provided in

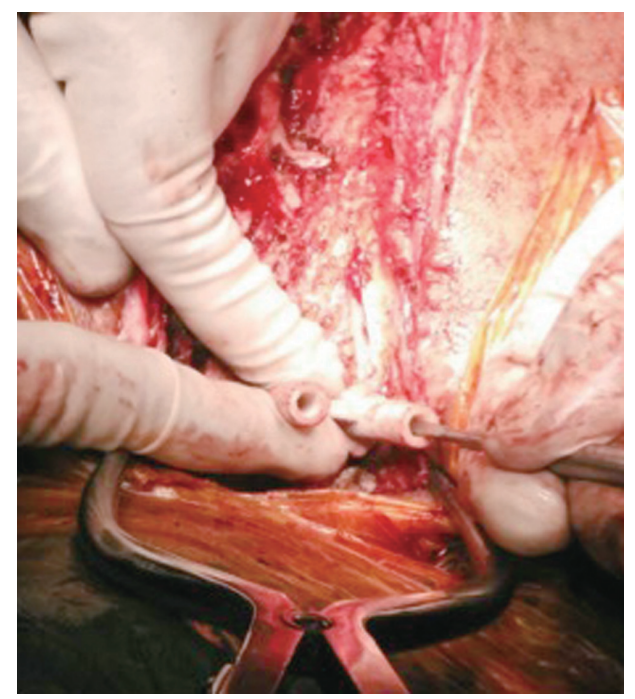

Fig. 1. Use of three-dimensional-printed polyurethane drill guide for inserting pedicle screws. informed consent for patients [29]. We have started using 3D-printed specific drill guides made of polyurethane in cases of deformity correction for inserting pedicle screws, particularly on the concave side of the curve (Fig. 1). We found that these jigs were also useful in cases of revision deformity correction wherein the native anatomy and bony landmarks were distorted because of index surgery (Fig. 2).

\section{Craniocervical Surgery}

Full-dimensional 3D-printed models have also been used to provide adequate rod contouring in 15 patients with rheumatoid arthritis who underwent craniocervical fusion [30]. 3D polyurethane models were fabricated using rapid prototyping techniques from 1-mm slice thickness individual CT scan. Using models as templates, appropriate shapes of plate rods were constructed in advance. A halo vest was used preoperatively to adjust the occipitocervical angle. A CT scan was performed in this position of comfort to obtain the image from which the 3D models were made. No patients had complaints of dysphagia following surgery.

In our clinical practice, customized 3D-printed models are widely used in the management and surgical planning of complicated spinal pathologies. One such example is the use of 3D-printed models for planning complicated surgeries of atlantoaxial dislocation with fixed kyphosis and an anomalous vertebral artery. Using axial CT scans, the $3 \mathrm{D}$ reconstruction of native anatomy was created (Fig. 3A, B). Later, an arterial gradient was added to recon-

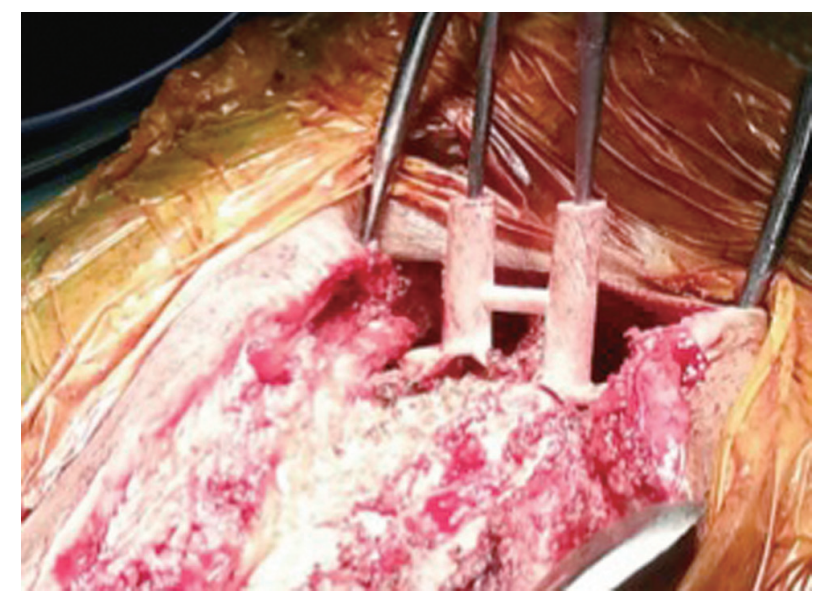

Fig. 2. Use of three-dimensional-printed polyurethane drill guide for inserting pedicle screws in a revision deformity correction case. 

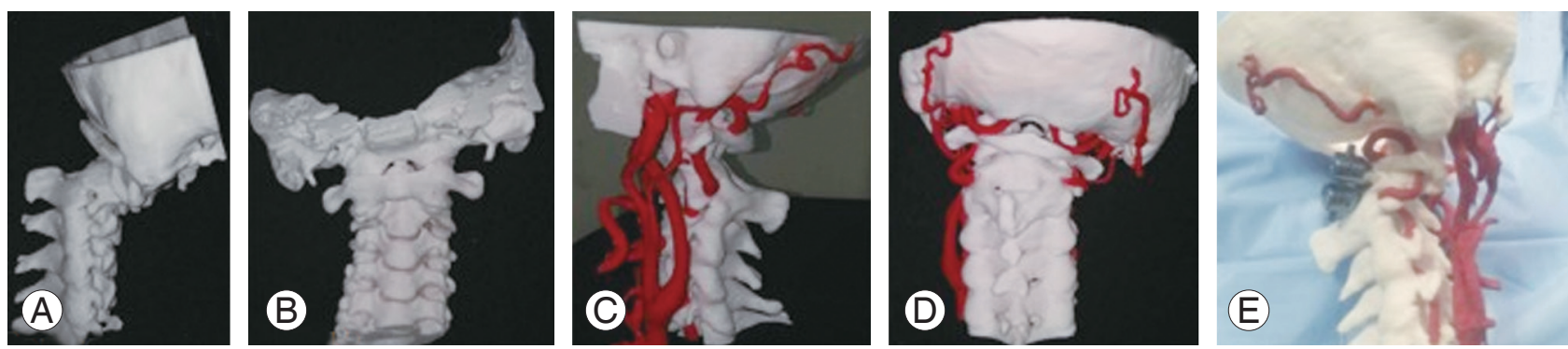

Fig. 3. Use of three-dimensional (3D)-printed models for planning complicated surgery of atlantoaxial dislocation with anomalous vertebral artery. (A, B) 3D reconstruction of native anatomy using axial computed tomography images. (C, D) 3D reconstruction of native anatomy with anomalous vertebral artery. (E) Proxy surgery performed on the 3D-printed model.

struct the native anatomy of the vertebral arteries (Fig. 3C, D). This data was then processed to make CAD files and eventually to print a 3D model. This model was used as a proxy for actual surgery in the preoperative period for planning the trajectory and dimensions of screws (Fig. $3 \mathrm{E})$.

\section{Interbody Fusion Devices}

3D printing technology has also been explored to produce interbody fusion devices. The material property of these interbody fusion devices has a vital role in good longterm functional outcome. An improperly designed device (cage) with faulty material can lead to stress shielding and subsidence [22]. It is desirable for an ideal cage to have the properties of native bone. Using 3D printing technology and bioengineering, biodegradable cages can be produced by having a Young's modulus similar to that of native cancellous bone [22]. However, a significant concern with using resorbable materials is their ability to carry loads both initially and as the material resorbs. If such a material resorbs too quickly before bone formation, there is a risk of disc space collapse due to material failure. In an animal study, Lamarca et al. demonstrated that the 3D-printed biodegradable cage plate system could provide sufficient mechanical load bearing to support typical cervical spine loads and maintain disc height throughout the 18-month experiment period [31]. The ability to integrate osteoconductive coating and/or osteoinductive factors such as BMP directly with a bioresorbable polymer surface can allow new fusion devices that better distribute and control release of osteoinductive factors in a 3D space while allowing better distribution of stresses at the bone-device interface to reduce subsidence and stress shielding.

\section{Disc Replacement Surgery}

The application of additive manufacturing to tissue bioengineering has stimulated the interest of researchers in manufacturing of bioengineered total disc replacement prostheses. At Cornell University in Ithaca, New York, Moriguchi et al. [32] created total disc replacement constructs that were manufactured using stem cell-infused bioink printers. These 3D-printed constructs contained cultured ovine nucleus pulposus cells in a central hydrogel that later regenerated themselves, while the circumferentially placed annulus fibrosus cells aligned the collagen matrix [32]. In a study of more than 100 rodents, disc height and biomechanical function were maintained for the entire life span of each rodent in the group that received the tissue-engineered discs [32].

\section{Minimally Invasive Spine Surgery}

In minimally invasive spine surgery, 3D-printed biomodels can be useful for determining whether the screws can be put in the confines of tubular retractor [28]. By measuring the soft tissue superficial to the spine, it is then possible to calculate the distance from the midline where the trajectories meet. Although the technology is still in trials, it is expected that it will not be too long before the implant-producing companies will be manufacturing patient-specific screw insertion sleeves for use in minimally invasive spine surgery [28].

\section{Constraints in the Use of Three-Dimensional Printing}

Even with the rapid progress in the technology of 3D printing, it is still not available universally. The cost of 
the entire manufacturing procedure for these $3 \mathrm{D}$ models depends on the material being used. During CT scanning, the patients should be properly immobilized to prevent motion artifacts, which disrupt the continuity in the spinal model [33]. To achieve a high degree of reproducibility, the distance between the CT cuts should not exceed $1.5 \mathrm{~mm}$ [34]. Pseudoforaminas are the defects in the image due to low CT values produced by thin bone. The pseudoforamina and metal artifacts caused by in situ implants must be corrected and eliminated [35]. It is sometimes not possible to produce real-size spinal models in one part. Thus, smaller parts are prepared by $3 \mathrm{D}$ printing and then assembled together by introducing minimal additional discrepancies relative to real anatomy [33]. These models depict only the vertebral column and not the surrounding soft tissue. It should be noted that the neural and vascular anatomy as well as the soft tissues must be considered before definitive planning of osteotomies and reconstruction. Another constraint in the use of $3 \mathrm{D}$ printing technology is that the total production time is approximately 6 weeks. Producing a spinal model may require approximately 12 days, and producing customized spinal implants may require approximately 4 weeks [33].

\section{Conclusions}

Despite recent rapid development and progress, the technology of 3D printing is still in its nascent phase. Although much research is being conducted worldwide to support its advantages and viability, it will still take many years before $3 \mathrm{D}$ printing has a widespread and significant clinical impact. Real-size 3D-printed biomodels made from polyurethane foam can be used to provide an excellent understanding of complex spinal pathologies that cannot be obtained from current imaging methods for highly selected patients with severe complex spinal pathologies. These models also provide visual and tactile feedback. The continuous and growing research on tissue bioengineering and its application in conjunction with additive manufacturing has provided new opportunities for the treatment of degenerative disc disease. Overall, the future of $3 \mathrm{D}$ printing in spine surgery is encouraging.

\section{Conflict of Interest}

No potential conflict of interest relevant to this article was reported.

\section{Acknowledgments}

We thank Mr. Devarsh Vyas and Ms. Firoza Kothari from Anatomiz3d LLP, Mumbai, India for providing Fig. 3.

\section{References}

1. Michalski MH, Ross JS. The shape of things to come: 3D printing in medicine. JAMA 2014;312:2213-4.

2. Wohlers TT. Wohlers report 2008: state of the industry: annual worldwide progress report. Fort Collins (CO): Wohlers Associates; 2008.

3. Rengier F, Mehndiratta A, von Tengg-Kobligk H, et al. 3D printing based on imaging data: review of medical applications. Int J Comput Assist Radiol Surg 2010;5:335-41.

4. Sun W, Starly B, Nam J, Darling A. Bio-CAD modeling and its applications in computer-aided tissue engineering. Comput Aided Des 2005;37:1097-104.

5. Hoy MB. 3D printing: making things at the library. Med Ref Serv Q 2013;32:94-9.

6. Dybuncio M. Woman receives world's first 3D printed jaw. CBS News [Internet]. 2012 Feb 6 [cited 2017 Oct 10]. Available from: https://www.cbsnews. com/news/woman-gets-worlds-first-3d-printed-jawtransplant/.

7. Bonda DJ, Manjila S, Selman WR, Dean D. The recent revolution in the design and manufacture of cranial implants: modern advancements and future directions. Neurosurgery 2015;77:814-24.

8. Kurtz SM, Devine JN. PEEK biomaterials in trauma, orthopedic, and spinal implants. Biomaterials 2007;28:4845-69.

9. Zopf DA, Hollister SJ, Nelson ME, Ohye RG, Green GE. Bioresorbable airway splint created with a threedimensional printer. N Engl J Med 2013;368:2043-5.

10. Gonfiotti A, Jaus MO, Barale D, et al. The first tissueengineered airway transplantation: 5-year follow-up results. Lancet 2014;383:238-44.

11. Klein GT, Lu Y, Wang MY. 3D printing and neurosurgery: ready for prime time? World Neurosurg 2013;80:233-5.

12. Zein NN, Hanouneh IA, Bishop PD, et al. Threedimensional print of a liver for preoperative planning in living donor liver transplantation. Liver Transpl 2013;19:1304-10.

13. McFarlane H. Success of first personalized, 3D-print- 
ed sternum implant procedure in USA. Spine News International [Internet]. 2017 Oct 19 [cited 2017 Oct 10]. Available from: https://spinalnewsinternational. com/3d-printed-sternum-implant-usa/.

14. Murphy SV, Atala A. 3D bioprinting of tissues and organs. Nat Biotechnol 2014;32:773-85.

15. Leberfinger AN, Ravnic DJ, Dhawan A, Ozbolat IT. Concise review: bioprinting of stem cells for transplantable tissue fabrication. Stem Cells Transl Med 2017;6:1940-8.

16. Xu T, Binder KW, Albanna MZ, et al. Hybrid printing of mechanically and biologically improved constructs for cartilage tissue engineering applications. Biofabrication 2013;5:015001.

17. Ringeisen BR, Pirlo RK, Wu PK, Boland T, et al. Cell and organ printing turns 15: diverse research to commercial transitions. MRS Bull 2013;38:834-43.

18. Hurson C, Tansey A, O'Donnchadha B, Nicholson P, Rice J, McElwain J. Rapid prototyping in the assessment, classification and preoperative planning of acetabular fractures. Injury 2007;38:1158-62.

19. Niikura T, Sugimoto M, Lee SY, et al. Tactile surgical navigation system for complex acetabular fracture surgery. Orthopedics 2014;37:237-42.

20. Gbureck U, Holzel T, Klammert U, Wurzler K, Muller FA, Barralet JE. Resorbable dicalcium phosphate bone substitutes prepared by $3 \mathrm{D}$ powder printing. Adv Funct Mater 2007;17:3940-5.

21. Ulbrich CB, Zavaglia CA, Leivas TP, Teixeira F. Medi$\mathrm{cal}$ application of rapid prototyping in orthopedics surgical planning. In: Bartolo PJ, de Lemos A, Tojeira A, et al. Innovative developments in virtual and physical prototyping. Boca Raton (FL): CRC Press; 2011. p.85-7.

22. Gibbs DM, Vaezi M, Yang S, Oreffo RO. Hope versus hype: what can additive manufacturing realistically offer trauma and orthopedic surgery? Regen Med 2014;9:535-49.

23. Cui X, Breitenkamp K, Finn MG, Lotz M, D'Lima DD. Direct human cartilage repair using threedimensional bioprinting technology. Tissue Eng Part A 2012;18:1304-12.

24. Reeves P. 3D printing industry report: 3D printing and additive manufacturing in the medical and healthcare marketplace. [place unknown]: 3D Printing Industry; 2013.
25. Cui X, Dean D, Ruggeri ZM, Boland T. Cell damage evaluation of thermal inkjet printed Chinese hamster ovary cells. Biotechnol Bioeng 2010;106:963-9.

26. Zimmermann G, Moghaddam A. Allograft bone matrix versus synthetic bone graft substitutes. Injury 2011;42 Suppl 2:S16-21.

27. Xiu P, Jia Z, Lv J, et al. Tailored surface treatment of 3D printed porous Ti6Al4V by microarc oxidation for enhanced osseointegration via optimized bone in-growth patterns and interlocked bone/implant interface. ACS Appl Mater Interfaces 2016;8:17964-75.

28. Bagaria V, Chaudhary K, Shah S. Technical note: 3D printing and developing patient optimized rehabilitation tools (PORT): a technological leap. Int J Neurorehabil 2015;2:175. https://doi.org/10.4172/23760281.1000175.

29. D'Urso PS, Williamson OD, Thompson RG. Biomodeling as an aid to spinal instrumentation. Spine (Phila Pa 1976) 2005;30:2841-5.

30. Mizutani J, Matsubara T, Fukuoka M, et al. Application of full-scale three-dimensional models in patients with rheumatoid cervical spine. Eur Spine J 2008;17:644-9.

31. LaMarca F, Flanagan CL, Tseng WJ, et al. A new resorbable integrated cervical plate/cage fusion device modified with osteoconductive coating or osteoinductive factors: preliminary results in a pre-clinical Yucatan minipig model [Internet]. Plymouth (MI): Tissue Regeneration Systems [cited 2017 Oct 10]. Available from: http://www.tissuesys.com/trs_media/publications/ Link\%203.pdf.

32. Moriguchi Y, Mojica-Santiago J, Grunert P, et al. Total disc replacement using tissue-engineered intervertebral discs in the canine cervical spine. PLoS One 2017;12:e0185716.

33. Van Dijk M, Smit TH, Jiya TU, Wuisman PI. Polyurethane real-size models used in planning complex spinal surgery. Spine (Phila Pa 1976) 2001;26:1920-6.

34. Woolson ST, Fellingham LL, Dev P, Vassiliadis A. Three dimensional imaging of bone from analysis of computed tomography data. Orthopedics 1985;8:1269-73.

35. Solar P, Ulm C, Lill W, et al. Precision of threedimensional CT-assisted model production in the maxillofacial area. Eur Radiol 1992;2:473-7. 